\title{
Keynote Session: Strategies for Prevention and Control of HPV-Associated Cancers
}

\author{
Presented by Lillian R. Kreppel; Shivan J. Mehta, MD, MBA, MSHP; Mark H. Sawyer, MD; Edward L. Trimble, MD, MPH; \\ Susan T. Vadaparampil, PhD, MPH; and moderated by Wui-Jin Koh, MD
}

\begin{abstract}
Infection with HPV is responsible for $5 \%$ of cancers, most of which are preventable with vaccination. Unfortunately, although vaccination rates are increasing in the United States, many adolescents remain unvaccinated due to a multifactorial set of barriers that are cultural, psychosocial, structural, and financial. At this Keynote session at the NCCN 2021 Virtual Annual Conference, a selection of expert panelists discussed efforts to improve vaccination rates.

J Natl Compr Canc Netw 2021;19(5.5):601-604 doi: 10.6004/jnccn.2021.5004
\end{abstract}

Infection with HPV is responsible for $5 \%$ of the world's cancers. ${ }^{1}$ In the United States, HPV is estimated to cause approximately 36,000 cancers in men and women each year, of which $70 \%$ to $90 \%$ are preventable through vaccination. ${ }^{2}$ However, many age-appropriate individuals are never vaccinated. In most other parts of the world, the HPV burden is even greater. This simple means of prevention continues to be hampered by many barriers, including psychosocial, cultural, structural, and financial. Concerted efforts are focused on eliminating these barriers and greatly increasing vaccination rates, as described by a multidisciplinary panel of speakers in a Keynote session at the NCCN 2021 Virtual Annual Conference.

\section{HPV Is Common and Ignored}

"Nearly all sexually active men and women get the virus at some point in their lives. Most of us have had it and have no clue this is happening," said Lillian R. Kreppel, Executive Director and Co-Founder of the HPV Alliance and a patient advocate and cancer survivor.

Although HPV is often cleared by the immune system, and most of the approximately 200 subtypes identified to date cause no disease, persistent infection and health risks can manifest in some people. Lower-risk subtypes (such as HPV6 and HPV11) cause warts on or around the genitals, anus, mouth, or throat, but high-risk subtypes (such as HPV16 and HPV18) cause cancers. Of all HPV-associated cancers, the greatest percentage in women is cervical (49\%), whereas in men it is oropharyngeal (81\%) (Figure 1).

According to some research, HPV causes virtually all cervical cancers, most anal cancers, the majority of cancers of the vagina, vulva, and penis, and a significant proportion of cancers of the oropharynx. ${ }^{2}$

Now that vaccines for HPV have been developed and are widely available, the speakers agreed that attention needs to be directed toward preventing HPV infection, which is more effective than treating its potential oncogenic consequences. "It's time to move to primary prevention," Ms. Kreppel said. "We know how to prevent these cancers from happening in the first place, so let's do it!"

However, they added that although vaccination could prevent approximately 30,000 cancers a year in the United States alone, ${ }^{2}$ reaching that goal requires some work. First the multifactorial barriers to immunization must be removed.

\section{Global Perspective: Primary Prevention of HPV-Associated Cancers}

Close to 600,000 new cases of cervical cancer were diagnosed worldwide in 2020, with approximately 300,000 deaths due to the disease, ${ }^{4}$ but progress is being made. In 2018, at the 71st World Health Assembly, WHO General Director, Dr. Tedros Adhanom Ghebreyesus, issued a "call to action" for the prevention and control of cervical cancer. Two years later, a panel of experts presented a strategic plan that was unanimously approved by 192 countries at the 73rd World Health Assembly, according to Edward L. Trimble, MD, MPH, Senior Advisor for HPV and Global Cervical Cancer Research and Control, National Cancer Institute/National Institutes of Health/Department of Health and Human Services.

The initiative gives the WHO a framework for eliminating cervical cancer globally as a public health 

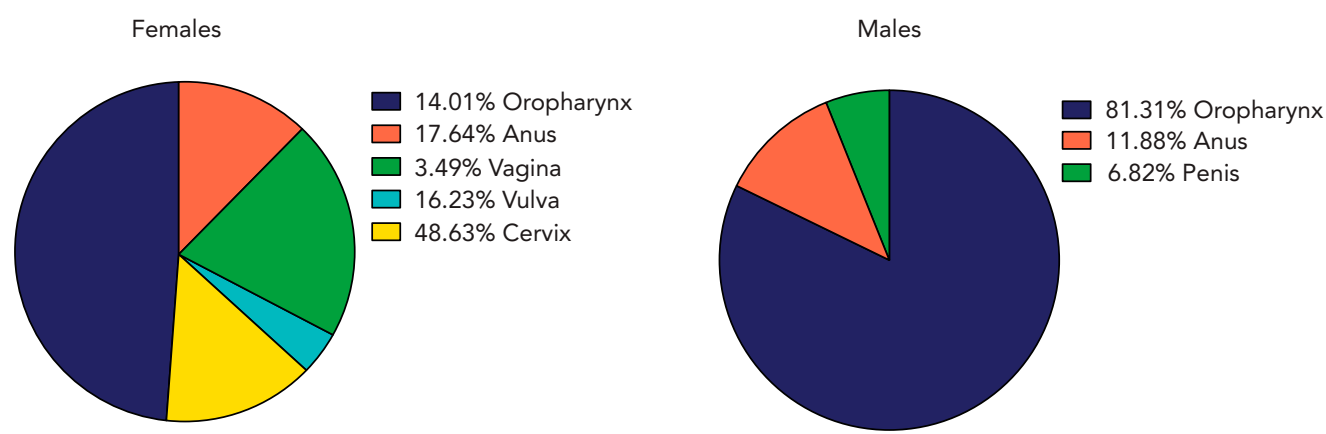

Figure 1. Incidence of HPV-associated cancers.

problem. It encompasses primary prevention of HPV infection, secondary prevention through screening and treatment of noninvasive neoplasia, multidisciplinary treatment of invasive disease, and palliative care.

Although in recent years the global HPV burden has decreased in some countries, especially the United States and Australia, it remains substantial in Latin America, Africa, Southern and Eastern Asia, and Eastern Europe. In fact, only 20 low- and middle-income countries have introduced HPV vaccination as part of national immunization programs, although many others have conducted pilot programs, reported Dr. Trimble.

This aside, strides have been made in primary prevention, fundamentally the development and approval of prophylactic HPV vaccines by Merck (Gardasil, Gardasil 9) and GlaxoSmithKline (Cervarix). Gardasil covers HPV6, 11, 16, and 18; Gardasil 9 covers those plus 5 additional subtypes; and Cervarix covers HPV16 and 18 and has a strong adjuvant that conveys cross-protection for more subtypes. These vaccines reduce incident infections by $>91 \%$ and prevent essentially $100 \%$ of persistent infections, Dr. Trimble said.

The vaccines rely on 2 to 3 doses given over 6 months, ideally before adolescents begin engaging in sexual activity. For persons not vaccinated under this protocol, "catch-up" vaccinations are recommended for women and men until 26 years of age; the FDA has recently expanded the indication for Gardasil 9 for women and men aged 27 to 45 years.

\section{Vaccination Rates Improving, But Problems Remain}

Vaccination rates have been improving, but they are not yet at the level of other standard pediatric vaccines. Although $\geq 90 \%$ of adolescents receive $\geq 1$ dose of Tdap (tetanus, diphtheria, pertussis), $\geq 1$ dose of meningococcal vaccine, and $\geq 2$ doses of MMR (measles, mumps, rubella), $74 \%$ of girls and $70 \%$ of boys received $\geq 1$ dose of HPV vaccine in 2019 and $59 \%$ and 52\%, respectively, received $\geq 2$ doses. ${ }^{5}$ The good news is that rates have increased steadily every year, "and we are approaching herd immunity," Dr. Trimble said.

The NCI and the Bill and Melinda Gates Foundation are now conducting a trial that randomizes individuals to 1 versus 2 doses of Gardasil 9 or Cervarix (ClinicalTrials.gov identifier: NCT03180034). Because a global shortage of HPV vaccines is predicted until 2025 (when a generic vaccine may become available), a 1-dose regimen would be helpful, and has been approved by the WHO Global Advisory Committee on Vaccine Safety for areas with limited supplies.

Cost is another challenge to widespread vaccination. The cost in the United States is $\$ 150$ per dose for 2 to 3 doses plus administration costs, but much lower costs have been negotiated in other countries. There is also a lack of established infrastructure for administering it, societal and parental resistance to the vaccine, and misinformation spread through social media. The COVID-19 pandemic has added another challenge by delaying all immunizations, Dr. Trimble reported.

Finally, there are psychosocial issues related to HPV, primarily because infected sites are linked to sexual behavior. From a patient and population perspective, according to Ms. Kreppel, the best way to reduce HPVrelated cancers is to destigmatize HPV infection. The message needs to get out, she said, that HPV infection results from skin-to-skin contact and is not the result of unsafe sex or promiscuity. Her organization is working to "lower the temp on the stigma" through campaigns with messages such as "HPV: no shame!"

\section{Insights From the Pediatrician's Perspective}

One aspect of HPV delivery that is not an issue is education of providers, according to Mark H. Sawyer, MD, Professor of Clinical Pediatrics, University of California San Diego School of Medicine and Rady Children's HospitalSan Diego. "Pediatricians and family practitioners are well educated about HPV vaccine and are doing a pretty good job recommending it," he said.

The problem is that their task is challenging. "Talking to parents of young adolescents and convincing 
them their kids will eventually have sex is a hard sell," he said. "They think they're liberating their adolescents to have sex." Continued propagation of myths and vaccine hesitancy are, he believes, "smokescreens for not wanting to confront the link that HPV has with sexuality."

"On top of that," he added, "the vaccine aims to prevent something that may not happen for 20 years. It's like asking a 30-year-old to start saving for retirement."

Physicians need quick and effective communication techniques to not only educate but also motivate parents and adolescents to seek vaccination, and their recommendation needs to be strong. They need to normalize HPV vaccination as yet another routine shot, perhaps by offering it at the same time as other vaccinations. And physicians need to emphasize that "we are out for the same outcome as the parents - the health of their child — and we want to prevent cancer in the future," Dr. Sawyer suggested.

Susan T. Vadaparampil, PhD, MPH, Associate Center Director of the Office of Community Outreach, Engagement and Equity, Moffitt Cancer Center, agreed that provider recommendation is central. Acknowledging that physicians and their staff have many competing demands for their attention and time, she is pushing for ways to "prime the community, patients, and parents to be receptive around messaging," she said.

Along these lines, it is important that vaccine advocates know their own communities: its needs and priorities, and where potential partnerships may exist. Such community partners can help "elevate the importance of vaccination and deliver the message broadly," Dr. Vadaparampil continued.

In Tampa, for example, she has aligned with the American Cancer Society on local initiatives, and on the state level she has a rotating list of partners to raise awareness around HPV Awareness Day on March 4. "I encourage all of us to think broadly about opportunities in the community," she said.

\section{Leveraging Insights From \\ Behavioral Economics}

Shivan J. Mehta, MD, MBA, MSHP, Associate Chief Innovation Office at Penn Medicine, and Assistant Professor of Medicine and Health Policy, Perelman School of Medicine, University of Pennsylvania, discussed how behavior can be leveraged to improve vaccine acceptance. He said the reason that vaccine rates have stalled at about $50 \%$ comes down to human behavior.

"Clinicians have to prescribe vaccines and encourage families and patients to have them, then the family and patients have to take up the offer and receive it," he said. "Conventional models of behavior focus on education. But we know that's not sufficient. You have to not only know about it but also show up to have it."
"Shortcuts" and "biases" help humans to make certain decisions more easily, Dr. Mehta said, but can also present problems. For example, "present time bias" makes it hard to think about a payoff in the future (ie, vaccination today, prevention of cancer in 20 years). The benefits are less tangible and they are far down the road. This is one factor.

Logistics is another factor. In a study from his institution, rates of hepatitis C screening among "baby boomers" significantly increased when eligible patients were directly sent a test request they could take straight to a laboratory-bypassing doctor's visits and so forth. He reported that rates doubled, from $19 \%$ to $43 \%{ }^{6}$

But perhaps the most actionable step, Dr. Mehta said, is to require families and adolescents to "opt out" rather than "opt in" for the vaccine. In other words, make vaccination the default option, which ensures it is easier to get the vaccine than to refuse it. "This still allows complete choice: it's just guiding people in the direction that is health-promoting."

\section{Ways to Make Vaccination Easier}

To simplify the vaccination process, one proposal that has been adopted by some states is to administer the shots within the school system. Another proposal, presented by Dr. Sawyer, would be to vaccinate children at a younger age (approximately 9 years) to "distance the concern of parents over early sexual activity," he said.

In addition to including an opt-out option, Dr. Mehta further proposed incorporating HPV vaccination into the electronic health record to ease prescribing for physicians.

Dr. Trimble emphasized the need for continued communication and myth-busting in general. "Much misinformation is spread through social media. Denmark and Japan saw annual rates fall from $90 \%$ to $1 \%$ because of this," he noted.

\section{Questions From the Moderator}

At the end of the session, Wui-Jin Koh, MD, Senior Vice President/Chief Medical Officer, National Comprehensive Cancer Network, led the presenters through additional questions.

Dr. Koh: Why is HPV vaccination not recommended for older adults? Would adults who have had an HPVassociated cancer benefit from it?

Dr. Sawyer: From a public health perspective, obviously vaccination is more effective before exposure and will prevent all the cancers HPV can cause. Many individuals become monogamous in their 20s, but if a person is continuing to be exposed to HPV, then sure-get the vaccine. 
Ms. Kreppel: I had anal cancer and I got the vaccine at age 52. My thought was that it could protect me from other harmful strains.

\section{Dr. Koh: What is needed to make a school-based program} accepted?

Dr. Vadaparampil: There are a few states where HPV vaccination is a school entry requirement, but it's a policy issue. Many states have taken a run with this with varying levels of success. Some have required that educational material at least be sent to the parents. It's tempting to think that this may be the solution, but it depends on the state.

Dr. Sawyer: The vaccine completion rate is the bigger problem: adolescents get the initial vaccine but often not the next. To avoid the initial decision about vaccinating in the schools, we could let the initial vaccination happen in the physician's office but give the second in the school. This might be a way for schools to ease into the concept because the decision was already made by the family.
Dr. Koh: Looking at HPV nonavalent vs bivalent vaccines, would the bivalent vaccine miss new strains for which the patient is not immunized?

Dr. Trimble: We think one does get more protection from nonavalent vaccines, but these require more energy to manufacture and are more expensive. The bivalent vaccine (Cervarix) comes with an excellent adjuvant and we have noted that it has some crossreactivity with, and therefore protection from, HPV subtypes other than HPV16 and 18. As we roll out HPV vaccines around the world, the bivalent vaccine is a reasonable alternative because of its excellent adjuvant. The big advance will be developing a generic to bring the cost down, which is especially important for low- and middle-income countries.

Disclosures: Dr. Vadaparampil disclosed serving on the speaker's bureau for GlaxoSmithKline and receiving grant support from Gilead. The remaining speakers have disclosed no relevant financial relationships.

Correspondence: Wui-Jin Koh, MD, National Comprehensive Cancer Network, 3025 Chemical Road, Suite 100, Plymouth Meeting, PA 19462 Email: Koh@nccn.org

\section{References}

1. Martel C, Plummer M, Vignat J, Franceschi S. Worldwide burden of cancer attributable to HPV by site, country and HPV type. Int J Cancer 2017;141:664-670

2. Saraiya M, Unger ER, Thompson TD, et al. US assessment of HPV types in cancers: implications for current and 9-valent HPV vaccines [published online April 29, 2015]. J Natl Cancer Inst.

3. Centers for Disease Control and Prevention. How Many Cancers Are Linked With HPV Each Year? Accessed March 21, 2021. Available at: https://www.cdc.gov/cancer/hpv/statistics/cases.htm
4. International Agency for Research on Cancer. Global Cancer Observatory. Accessed March 21, 2021. Available at: https://gco.iarc.fr/

5. Elam-Evans LD, Yankey D, Singleton JA, et al. National, regional, state and selected local area vaccination coverage among adolescents aged 13-17 years-United States, 2019. Centers for Disease Control and Prevention. MMWR Morb Mortal Wkly Rep 2020;69:1109-1116.

6. Mehta SJ, Day SC, Norris AH, et al. Behavioral interventions to improve population health outreach for hepatitis $\mathrm{C}$ screening: randomized clinical trial [published online May 18, 2021]. BMJ doi: https://doi.org/10.1136/bmj.n1022. 